\title{
Some alternate models for nearly constant loss in conductive systems
}

\author{
J. Ross Macdonald* \\ Department of Physics and Astronomy, University of North Carolina, Chapel Hill, North Carolina 27599-3255
}

(Received 4 December 2001; revised manuscript received 30 May 2002; published 20 August 2002)

\begin{abstract}
The qualitative serial-response approach recently suggested by León, Rivera, and co-authors for describing nearly constant loss (NCL) in conductive systems proposes that NCL arises entirely from vibrating ions confined in cages by potential barriers. Their identification of the cage potential-well activation energy as that of single ions and also as that of the thermally activated crossover between hopping and NCL behavior is inconsistent with prior identification of the single-ion energy in the Ngai coupling model, casting doubt on the physical basis of the serial approach. Its authors suggested that their experimental data, showing hopping and NCL behavior, could not be described by means of a parallel (sum) combination of expressions describing these two processes. Here, using essentially exact synthetic data of the same character as the experimental frequency-response data of these authors, it is demonstrated that either a parallel or a series complex constantphase response element (CPE) can lead to NCL results similar to theirs with a crossover between hopping and NCL response not of exact Arrhenius form. A plausible alternate to the serial NCL model is discussed. It involves a quantitative parallel CPE model that identifies NCL frequency response as being primarily a bulk-dielectric phenomenon arising from interactions between oscillating mobile charge carriers and the dipoles of the bulk material.
\end{abstract}

DOI: 10.1103/PhysRevB.66.064305

\section{INTRODUCTION}

Recently, much work has been devoted to trying to understand the origin of the nearly-frequency-independent part of the dielectric loss present in all ionic conductors and dominant at sufficiently low temperatures and/or high frequencies. ${ }^{1-6}$ Such behavior leads, when the data are transformed to the complex conductivity level, to frequency response of the real part of the complex conductivity, $\sigma^{\prime}(\omega)$, that depends linearly or nearly linearly ${ }^{2,6}$ on frequency, depending on whether the loss is taken as constant (CL) or nearly constant (NCL). When the experimental loss is very close to being frequency independent, the CL-NCL distinction can only be established by detailed fitting of the data (see, e.g., Refs. 1, 3, and 4). Ngai has recently characterized such loss as ubiquitous in ion conducting glasses, melts, and crystals and as a spectacular phenomenon. ${ }^{1}$ Since its detailed provenance is still doubtful, ${ }^{1,2,4,6}$ it is particularly important to attempt to elucidate as many aspects of it as possible.

In earlier work of Nowick and his collaborators, CL was characterized as a universal phenomenon, ${ }^{7}$ and a response form equivalent to

$$
\sigma^{\prime}(\omega)=\sigma_{0}\left[1+\left(\omega / \omega_{0}\right)^{n}\right]+A \omega
$$

was proposed, ${ }^{8,9}$ with $0<n<1$ and both $\sigma_{0}=1 / \rho_{0}$, the dc conductivity, and $\omega_{0}$ thermally activated. Here the first term in Eq. (1), modeling long-range ionic motion, has often been termed universal dynamic response and credited to Jonscher, $1973-1978,{ }^{1,2,6}$ but the complex generalization of this term, a Cole-Cole expression defined at the complex resistivity level, the ZC model, was used by Schwan for conductivesystem analysis as early as 1957 (see Ref. 10). Further, the ZC model has been shown to be less appropriate for describing resistivity dispersion than several other models, particu- larly a fully complex Kohlrausch-William-Watts expression, previously identified as the KWW1 model and hereafter designated by $\mathrm{K} 1 .{ }^{4,10}$

The second term in Eq. (1), with the parameter $A$ slowly increasing with increasing temperature, ${ }^{1}$ represents CL. Later work suggested that although CL was often a good approximation, characterization of relevant experimental data as NCL was more appropriate. ${ }^{3-5,11}$ Further, no exact expression for the imaginary response corresponding to the real part $A \omega$ is known, and it has been suggested that CL over an appreciable frequency range is nonphysical. ${ }^{3}$

Although most prior NCL work has dealt only with $\sigma^{\prime}(\omega)$ response, it is more appropriate to consider fitting models that incorporate both real and imaginary response. A plausible expression for modeling NCL is of complex powerlaw form and may be expressed as either a constant-phase element CPE in parallel (PCPE) with ionic low-frequency dispersive response or one in series (SCPE) with it. ${ }^{3-5}$ Appropriate CPE expressions are listed in the caption of Table I, and NCL requires that both the $\gamma_{\mathrm{PC}}$ and $\left(1-\gamma_{\mathrm{SC}}\right)$ exponents be less than about 0.1 , while the CL condition, $\gamma_{\mathrm{PC}}=0$, yields zero loss. The general CPE complex power law, $\sigma_{\mathrm{CPE}}=B(i \omega)^{m}$, where $B$ is frequency independent and 0 $<m \leqslant 1$, has been widely used in immittance spectroscopy as a model for data fitting. ${ }^{12}$ For example, it is the frequencydependent part of the ZC model, and CPE response has been ascribed to many diverse physical processes, such as fractal behavior, a distribution of relaxation times, electrode roughness, etc. ${ }^{10,12}$ Here it will be used to represent either NCL or electrode effects, as discussed in more detail in Ref. 3.

References 2 and 6 present important, ground-breaking data on the crystalline fast ionic conductor, $\mathrm{Li}_{0.18} \mathrm{La}_{0.61} \mathrm{TiO}_{3}$, data that show low-temperature NCL behavior and a crossover at sufficiently high temperatures to power-law frequency dependence. The authors of these papers explicitly state that their work is not intended to develop a theory of 
TABLE I. Nearly-constant-loss quantities for PK1- and K1S-model data and fits. The parallel PCPE is given by $\sigma_{\mathrm{PC}}(\omega)$ $\equiv \varepsilon_{V} A_{\mathrm{PC}}(i \omega)^{1-\gamma_{\mathrm{PC}}}$ and the series SCPE by $\sigma_{\mathrm{SC}}(\omega) \equiv \varepsilon_{V} A_{\mathrm{SC}}(i \omega)^{\gamma_{\mathrm{SC}}}$. We use $A_{X \mathrm{C}}=A_{X 0} \exp \left(-E_{X \mathrm{C}} / k T\right)$ and $A_{X \mathrm{C}}=B_{X 0} \exp \left(b_{X \mathrm{C}} T\right)$, where $X=\mathrm{P}$ or $\mathrm{S}$. $S_{\mathrm{F}}$ is the relative standard deviation of a fit. $S_{\mathrm{Fb}}$ is that for fits of $A_{X \mathrm{C}}(T)$ using the $b_{X \mathrm{C}}$ parameter, $S_{\mathrm{F} E}$ is that using $E_{X \mathrm{C}}$, and $S_{\mathrm{FC}}$ is that for Arrhenius fits of the crossover radial frequency $\omega_{\mathrm{CO}}(T)$, yielding the estimates of $E_{\omega \mathrm{CO}}$ shown below. See Fig. 1 and the text for discussion of the results in columns 5 and 7.

\begin{tabular}{|c|c|c|c|c|c|c|}
\hline $\begin{array}{l}1 \\
T(\mathrm{~K}) \\
E(\mathrm{eV}) \\
100 S_{\mathrm{F}}\end{array}$ & $\begin{array}{c}2 \\
\mathrm{PK} 1 \\
E_{\mathrm{PC}}=0.00 \\
A_{\mathrm{PC}}\end{array}$ & $\begin{array}{c}3 \\
\mathrm{PK} 1 \\
E_{\mathrm{PC}}=0.06 \\
A_{\mathrm{PC}}\end{array}$ & $\begin{array}{c}4 \\
\mathrm{PK} 1 \\
E_{\mathrm{PC}}=0.09 \\
A_{\mathrm{PC}}\end{array}$ & $\begin{array}{c}5 \\
\mathrm{~K} 1 \mathrm{~S} \\
E_{\mathrm{PC}}=0.06 \\
A_{\mathrm{SC}}\end{array}$ & $\begin{array}{c}6 \\
\mathrm{PK} 1 \\
b_{\mathrm{PC}}=0.015 \\
A_{\mathrm{PC}}\end{array}$ & $\begin{array}{c}7 \\
\mathrm{PK} 1 \\
b_{\mathrm{PC}}=0.030 \\
A_{\mathrm{PC}}\end{array}$ \\
\hline $183 \mathrm{~K}$ & 400 & 843.44 & 1224.8 & 1539.3 & 627.35 & 878.30 \\
\hline $153 \mathrm{~K}$ & 400 & 400 & 400 & 553.55 & 400 & 358.65 \\
\hline $127 \mathrm{~K}$ & 400 & 157.56 & 98.89 & 179.77 & 270.83 & 165.03 \\
\hline $100 S_{\mathrm{F} b}$ & & 12.1 & 17.8 & 10.2 & & \\
\hline $100 S_{\mathrm{FE}}$ & & & & 5.58 & 6.31 & 12.7 \\
\hline$E_{\omega \mathrm{CO}}$ & $0.363 \pm 0.002$ & $0.191 \pm 0.002$ & $0.106 \pm 0.002$ & $0.191 \pm 0.002$ & $0.277 \pm 0.010$ & $0.193 \pm 0.016$ \\
\hline $100 S_{\mathrm{FC}}$ & 3.82 & 3.84 & 3.85 & 3.84 & 20.5 & 35.0 \\
\hline$E_{\sigma \mathrm{CO}}$ & 0.363 & 0.251 & 0.196 & 0.251 & 0.307 & 0.252 \\
\hline
\end{tabular}

NCL but to present important experimental facts that may guide others to construct such models. ${ }^{6}$ Their detailed analysis of their data has led them, however, to several potentially important conclusions. The purpose of the present work is to test the appropriateness of these conclusions by generating essentially exact synthetic frequency- and temperatureresponse data sets of the same character as those presented in Refs. 2 and 6, then analyze them in the same ways as employed in these references, and consider their implications for a new physical NCL model. ${ }^{4}$

The conclusions in question are the following:

(i) "The experimental findings suggest a serial picture in which NCL is automatically terminated when the ions have a significant probability to leave their cages and start hopping between adjacent wells." 6 Before this happens, the ions are assumed to be vibrating in their cages.

(ii) "The ac conductivity cannot be described by the 'augmented Jonscher expression' in the whole temperature and frequency range." Thus, a composite parallel-response model consisting of the sum of power-law response and a CL term, as in Eq. (1), is "at variance with the observed temperature dependence of the NCL over a wide temperature range."

(iii) The crossover between the hopping and NCL regimes is "thermally activated with an activation energy $E$ $=0.17 \mathrm{eV}$, significantly lower than that observed for the $\mathrm{dc}$ conductivity (at low temperatures), $E=0.4 \mathrm{eV}$."

(iv) The above smaller activation energy "has the same value as that obtained from NMR spin-lattice relaxation," equals the height of the single-ion potential well, $E_{m}$, and is also essentially equal to the activation energy of the hightemperature part of the dc conductivity. Note that the singleion potential barrier is also defined as that of the hightemperature primitive response of the Ngai coupling model. ${ }^{13}$

(v) The authors' picture suggests that "the mean-square displacement of ions while still caged would not be constant but would increase very slowly with time, providing a possible explanation of the NCL ...."

In Sec. II, data generation approaches are discussed and illustrated, Sec. III presents detailed crossover results for both parallel and series NCL approaches, and Sec. IV compares the serial NCL approach with a physically reasonable bulk-dielectric parallel one.

\section{DATA GENERATION CONSIDERATIONS}

The authors of Refs. 2 and 6 plotted their $\sigma^{\prime}(\omega, T)$ data in isochronal format: six constant-frequency curves vs temperature $T$ (or $1000 / T$ ). They also calculated six crossover temperatures between the low-temperature NCL response (taken as CL) and an apparent fractional power-law response, such as that associated with Eq. (1) when $\left(\omega / \omega_{0}\right)^{n} \gg 1$. Isothermal results were later presented involving frequency-response curves for the three fixed temperatures 183,153 , and $127 \mathrm{~K}^{6}$ Crossover frequencies, $\omega_{\mathrm{CO}}$, marking a transition from lowfrequency ionic-hopping dispersion to apparent CL behavior, were obtained from the $10 \%$ criterion $\sigma^{\prime}\left(\omega_{\mathrm{CO}}\right)=1.1 A \omega_{\mathrm{CO}}$. They led to $\sigma^{\prime}\left(\omega_{\mathrm{CO}}\right)$ results in good agreement with those estimated from the crossover temperatures. In particular, the calculated crossover thermal activation energy $E_{\sigma \mathrm{CO}}$ was quoted as $0.17 \pm 0.03 \mathrm{eV}$. Although somewhat similar isochronal and isothermal plots appear in Ref. 14, the demonstration in Refs. 2 and 6 that there seems to be a thermally activated crossover whose activation energy may be estimated and physically identified is particularly important and is further discussed in the following section.

In order to obtain synthetic data to approximate that in Refs. 2 and 6, it is necessary to pick a response model and determine appropriate values for its parameters. Because the $\mathrm{K} 1$ has been shown to be a particularly appropriate fitting model, ${ }^{3-5,10}$ I shall begin with the PK1 model, a parallel combination of the K1 low-frequency conductive-system dispersion model and the PCPE, usually defined at the complex dielectric constant level but expressed at the conductiv- 


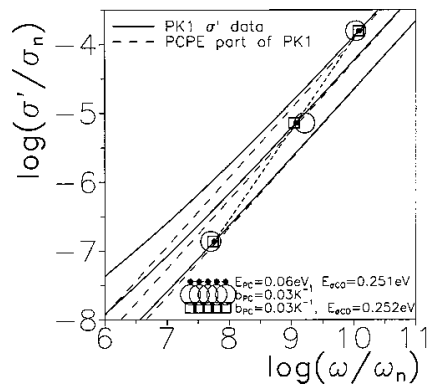

FIG. 1. Frequency dependence of conductivity data calculated from the PK1 conductive-system response model for three values of temperature: 183,153 , and $127 \mathrm{~K}$, from the top to the bottom. The values of the nearly-constant-loss PCPE parameters used are $\gamma_{\mathrm{PC}}$ $=10^{-4}$, taken to be temperature independent, and the three values of $A_{\mathrm{PC}}$ shown in columns 3 and 7 of Table I, where definitions of parameters are listed. The $\mathrm{K} 1$ parameter values are discussed in Sec. II. Filled circle symbols designate PK1 crossover points for $10 \%$ deviation from the PCPE lines for the $E_{\mathrm{PC}}=0.06-\mathrm{eV}$ (column 3) data, while open-circle symbols show those for the $b_{\mathrm{PC}}$ $=0.030-\mathrm{K}^{-1}$ (column 7) choice. The open-square points were obtained from an Arrhenius fit of the $b_{\mathrm{PC}}=0.030-\mathrm{K}^{-1}$ crossover $\sigma^{\prime}\left(\omega_{\mathrm{CO}}, T\right)$ values. The quantities $\omega_{n}=1 \mathrm{rev} / \mathrm{s}$, and $\sigma_{n}=1 \mathrm{~S} / \mathrm{cm}$.

ity level in the caption of Table I. The quantity $\varepsilon_{V}$ is the permittivity of vacuum.

The K1 model involves the parameters $\rho_{0}, \beta_{1}$, and $\tau_{o}$, where $\tau_{o}$, the characteristic relaxation time of the model, is thermally activated with an activation enthalpy of $E_{\tau}$, and $\beta_{1}$ is the shape parameter. Some numerical experimentation for $T=153 \mathrm{~K}$ led to the $\mathrm{K} 1$ values $\rho_{0}=1 / \sigma_{0}=10^{17} \Omega \mathrm{cm}$ and $\tau_{o}=10^{7} \mathrm{~s}$. In addition, the value of $\beta_{1}$ was selected as 0.35 and taken to be temperature independent. ${ }^{4}$ Although the K1 model cannot be expressed analytically for arbitrary $\beta_{1}$, it can be accurately calculated numerically using the complex-nonlinear-least-squares-fitting program LEVM. ${ }^{15}$

Here I shall follow common usage and take $\sigma_{0} T$ as involving the same thermal activation energy as that of $\tau_{o}$ $=\tau_{o} \exp \left(E_{\tau} / k T\right)$, where $\tau_{o}$ is independent of temperature. In contrast, although in Ref. 6 the Nernst-Einstein equation ${ }^{16}$ was found to be applicable, the authors took $E_{\sigma}=E_{\tau}$ (see Ref. 17) rather than the present $E_{\sigma T}=E_{\tau}$ choice, where $E_{\sigma T}$ is that for $\sigma_{0} T$ instead of that of $\sigma_{0}$. Recent fitting results for $0.88 \mathrm{ZrO}_{2} \cdot 0.12 \mathrm{Y}_{2} \mathrm{O}_{3}$ data $^{4}$ and other materials ${ }^{18-22}$ show that $\beta_{1}$ is indeed virtually temperature independent and although $E_{\sigma T}=E_{\tau}, E_{\sigma} \neq E_{\tau}$.

In Ref. 6, the authors quote a low-temperature-region value of $E_{\sigma} \simeq E_{\tau}=0.4 \mathrm{eV}$ for their $\mathrm{Li}_{0.18} \mathrm{La}_{0.61} \mathrm{TiO}_{3}$ material. Using this value for $E_{\sigma T}$ and $E_{\tau}$ in the above relations allows one to calculate the consistent values of $\tau_{o}$ and $\rho_{0}$ for 183 and $127 \mathrm{~K}$. To obtain the response of the composite PK1 model at all three temperatures, we must finally specify values of the PCPE $A_{\mathrm{PC}}$ and $\gamma_{\mathrm{PC}}$ parameters. In order to approximate the CL assumption in Refs. 2 and 6, we take a temperature-independent value of the NCL quantity $\gamma_{\mathrm{PC}}$ of $10^{-4}$. At $153 \mathrm{~K}$, we set $A_{\mathrm{PC}}=400$; then when values of the $E_{\mathrm{PC}}$ or $b_{\mathrm{PC}}$ parameters of $A_{\mathrm{PC}}$ (see caption of Table I) are selected, values of $A_{\mathrm{PC}}$ at the other two temperatures may be calculated.
PK1-model responses for the above choices and $E_{\mathrm{PC}}$ $=0.06 \mathrm{eV}$ are shown in Fig. 1 for all three temperatures. These curves, calculated with LEVM, very closely resemble the experimental ones presented in Ref. 6, with, however, a somewhat different frequency scale. It needs to be emphasized, however, that they arise here from a composite parallel, not series or serial-, response models. The dashed lines allow one to compare the total response with that associated only with PCPE contributions. The crossover points in the figure are discussed in detail in the next section. Clearly, these Fig. 1 results disagree with conclusion (ii) listed in Sec. I.

\section{CROSSOVER ANALYSIS}

\section{A. PK1-model data generation}

In order to explore crossover behavior, many sets of synthetic data have been generated and their crossover characteristics calculated. Some of these results are presented in Table I. For PK1 parallel-response situations, the K1-model parameter values were taken to be fixed and the effects of various temperature dependencies of $A_{\mathrm{PC}}$ were investigated. The data sets involved 50 points per decade, and the PK1 crossover frequency $\omega_{\mathrm{CO}}$ was determined as follows. First, the ratio $r=\sigma^{\prime}(\omega) / \sigma_{\mathrm{PC}}^{\prime}(\omega)$ was calculated over the entire frequency range. Then, for a particular choice of this ratio, such as 1.1, interpolation with five or more points was used to obtain a value of $\omega_{\mathrm{CO}}$ accurate to four or more significant figures. At crossover, $\sigma^{\prime}\left(\omega_{\mathrm{CO}}\right)=r \sigma_{\mathrm{PC}}^{\prime}\left(\omega_{\mathrm{CO}}\right)$ $=r \varepsilon_{V} A_{\mathrm{PC}} \sin \left(\pi \gamma_{\mathrm{PC}} / 2\right) \omega_{\mathrm{CO}}^{1-\gamma_{\mathrm{PC}}}$. Therefore, if $\omega_{\mathrm{CO}}$ is taken to be thermally activated with activation energy $E_{\omega \mathrm{CO}}$, it will contribute a term $\left(1-\gamma_{\mathrm{PC}}\right) E_{\omega \mathrm{CO}}$ to $E_{\sigma \mathrm{CO}}$, the activation energy associated with $\sigma^{\prime}\left(\omega_{\mathrm{CO}}\right)$.

\section{B. PK1-model crossover results}

Although I concentrate on using the parallel PCPE model here, some attention is given to $\mathrm{K} 1 \mathrm{~S}$ response ( $\mathrm{K} 1$ and $\mathrm{SCPE}$ in series at the complex resistivity level) in order to illustrate some of its similarities and differences from those of the PK1 model. First, notice in Table I that for columns 2-4, where $A_{\mathrm{PC}}$ is taken thermally activated, $E_{\sigma \mathrm{CO}}=\left(1-\gamma_{\mathrm{PC}}\right) E_{\omega \mathrm{CO}}$ $+E_{\mathrm{PC}}$ and the relative standard deviations of $E_{\sigma \mathrm{CO}}$ and $E_{\omega \mathrm{CO}}$, and $S_{\mathrm{F} \sigma \mathrm{C}}$ and $S_{\mathrm{F} \omega \mathrm{C}}$, are thus essentially the same. Therefore, only values of $S_{\mathrm{F} \omega \mathrm{C}} \equiv S_{\mathrm{FC}}$ are listed in the table. For the present choice of $\gamma_{\mathrm{PC}},\left(1-\gamma_{\mathrm{PC}}\right) E_{\omega \mathrm{CO}}$ may be replaced by $E_{\omega \mathrm{CO}}$ to good approximation. Column 2 of the table, for the $E_{\mathrm{PC}}=0$ situation, involves temperatureindependent values of $A_{\mathrm{PC}}$ and leads to the surprising result that

$$
E_{\sigma \mathrm{CO}}=E_{\omega \mathrm{CO}}=\delta \equiv E_{\sigma T}-\Delta \neq E_{\sigma T}=0.4 \mathrm{eV} .
$$

Here $\Delta \cong 0.0371 \mathrm{eV}$, a significant quantity not identified in the work of Refs. 2 and 6.

For the present $E_{\mathrm{PC}}=0$ situation, in Ref. 6 it is assumed that $E_{\sigma \mathrm{CO}}=E_{\sigma}=0.4 \mathrm{eV}$. Although it might be plausible herein to calculate $E_{\sigma \mathrm{CO}}$ from $T \sigma^{\prime}\left(\omega_{\mathrm{CO}}, T\right)$ values, rather 
than from $\sigma^{\prime}\left(\omega_{\mathrm{CO}}, T\right)$ ones, the latter choice has been made in order to compare more directly with the results of Refs. 2 and 6. Further, it turns out that values calculated with the former choice all exceed the latter-choice ones by only 0.013 $\mathrm{eV}$, close to the value predicted from a result of Nowick, Vaysleyb, and Liu, ${ }^{16}$ and thus allowing simple conversion if desired.

Incidentally, the nonzero value of $\Delta$ found here does not arise from the use of the K1 model instead of the universal dynamic response part of Eq. (1). In the present frequency and temperature ranges, the $\sigma^{\prime}(\omega)$ response of the latter model is dominated by $\sigma_{0}\left(\omega / \omega_{0}\right)^{n}$ while that of the K1 becomes proportional to $\sigma_{0}\left(\omega \tau_{0}\right)^{1-\beta_{1}}$, an equivalent power law. Numerical experimentation shows that although the value of $\Delta$ is independent of the choice of $E_{\sigma T}$, and although the value of $\delta$ is independent of that of $A_{\mathrm{PC}}, \delta$ does satisfy $\delta\left(\beta_{1}-\gamma_{\mathrm{PC}}\right) \cong 0.12698 \mathrm{eV}$, at least for $10^{-4} \leqslant \gamma_{\mathrm{PC}} \leqslant 10^{-2}$. Further, it is clear that when $\gamma_{\mathrm{PC}}=\beta_{1}$, not a NCL situation, there is no crossover because the high-frequency-limiting slopes of $\sigma^{\prime}(\omega)$ associated with the K1, and the PCPE models are then identical.

Column 6 of Table I shows results for the $b_{\mathrm{PC}}$ $=0.015 \mathrm{~K}^{-1}$ choice. Although the $S_{\mathrm{FC}}$ value is very large, indicating a poor fit, the estimated $E_{\mathrm{PC}}$ value was 0.0298 $\pm 0.0033 \mathrm{eV}$, numerically nearly twice the input $b_{\mathrm{PC}}$ value. The column $7 A_{\mathrm{PC}}$ values were obtained by fitting the column $3 A_{\mathrm{PC}}$ values with its expression involving $b_{\mathrm{PC}}$, as defined in the caption of the table. Again the $S_{\mathrm{F} \omega \mathrm{C}} \equiv S_{\mathrm{FC}}$ value is very poor, and the resulting $E_{\mathrm{PC}}$ value was 0.0593 $\pm 0.0070 \mathrm{eV}$, once more about twice the input $b_{\mathrm{PC}}$ value. As shown in Fig. 1, the open-circle $b_{\mathrm{PC}}=0.030-\mathrm{K}^{-1}$ results fail to agree well with the filled-circle $E_{\mathrm{PC}}=0.06-\mathrm{eV}$ ones of column 3 and involve the extreme value of $S_{\mathrm{FC}}=0.35$. But note that even the filled-circle points, involving $S_{\mathrm{F} \sigma \mathrm{C}} \cong S_{\mathrm{F} \omega \mathrm{C}}$ $=0.04$, do not represent an exact Arrhenius response, although deviations are not evident on the log-log plot of Fig. 1. Thus, only inexact Arrhenius crossover behavior is found, raising doubts about the appropriateness of conclusion (iii).

The results shown in Table I for the PK1 model with $E_{\mathrm{PC}} \geqslant 0 \mathrm{eV}$ satisfy very closely the relations

$$
E_{\omega \mathrm{CO}}=\left(E_{\sigma T}-\Delta\right)-E_{\mathrm{PC}} /(1-n) \mathrm{eV}
$$

and

$$
E_{\sigma \mathrm{CO}}=\left(E_{\sigma T}-\Delta\right)-E_{\mathrm{PC}}(n / 1-n) \mathrm{eV},
$$

where $n=1-\beta_{1}$. For $b_{\mathrm{PC}} \neq 0$ situations, however, the case investigated in Ref. 6, the authors started with Eq. (1) of the present work and derived the expression

$$
E_{\sigma \mathrm{CO}}=E_{\sigma}-(n / 1-n) k b_{\mathrm{PC}} T^{2} \mathrm{eV} .
$$

If we use $E_{\sigma}=0.4-0.013=0.387 \mathrm{eV}, n=0.65$, and $b_{\mathrm{PC}}$ $=0.030 \mathrm{~K}^{-1}$, and take $T$ as the mean of the present three temperatures, Eq. (5) yields $E_{\sigma \mathrm{CO}} \simeq 0.273 \mathrm{eV}$, compared to the actual column 7 result of $0.252 \mathrm{eV}$. If instead we replace the $E_{\sigma}$ in Eq. (5) by $\left(E_{\sigma T}-\Delta\right)=0.3629 \mathrm{eV}$, as in Eq. (2), we obtain $0.249 \mathrm{eV}$. Also if we use Eq. (4) with $E_{\mathrm{PC}}$ replaced by

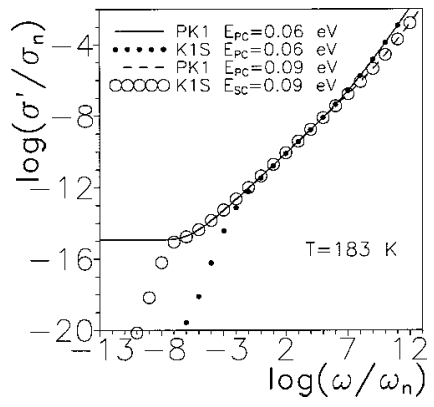

FIG. 2. Log-log exact $\sigma^{\prime}(\omega)$ responses for the parallel PK1 model and the series K1S one at $183 \mathrm{~K}$. See columns 3 and 4 of Table I for the PK1-model situations and column 5 for the $E_{\mathrm{PC}}$ $=0.06-\mathrm{eV}$ K1S one. The K1S model parameters were obtained by fitting PK1 data over the range from $10^{5}$ to $10^{11} \mathrm{r} / \mathrm{s}$, and the PK1 and K1S models were then used to calculate the present extrapolated-range responses.

the numerical value of $2 b_{\mathrm{PC}}$, we obtain the proper value of $0.252 \mathrm{eV}$. It is evident that $\Delta$ plays an important role in determining values of $E_{\sigma \mathrm{CO}}$.

It is worth mentioning what happens if one alternatively defines crossover as occurring at an abrupt transition from $\mathrm{K} 1$ response to PCPE response, similar in philosophy to that of the Ngai coupling model, but not consistent with the present simulation data. Then we may write $\sigma_{\mathrm{K} 1}^{\prime}\left(\omega_{\mathrm{CO}}\right)$ $=\sigma_{\mathrm{PC}}^{\prime}\left(\omega_{\mathrm{CO}}\right) \equiv \sigma^{\prime}\left(\omega_{\mathrm{CO}}\right)$, and, although the values of $\omega_{\mathrm{CO}}$ and $\sigma\left(\omega_{\mathrm{CO}}\right)$ are then found for the present data to be quite different from those following from the usual crossover definition, values of both $E_{\omega \mathrm{CO}}$ and $E_{\sigma \mathrm{CO}}$ are the same for both definitions.

Although the authors of Refs. 2 and 6 found that NCL temperature dependence was better described by exponential response involving $b T$, as defined in the caption of Table I, than by thermally activated response, the Table I results show that the latter response leads to about a ten-times closer approximation to Arrhenius crossover behavior than does the former.

\section{K1S-model crossover results}

The wide-range results of Fig. 2 show that although at sufficiently low frequencies it should be possible to distinguish between series and parallel NCL behavior, the close equality of these responses over four or more decades, as in the figure, renders such discrimination difficult at higher frequencies. Therefore, even though PCPE response is usually much more appropriate for most NCL situations, ${ }^{3,4}$ series response needs some investigation as well. Further, in actual situations with blocking or nearly blocking electrodes, it may be necessary to use a composite model such as PK1S, with the SCPE representing electrode effects and the PCPE one NCL ones. A series electrode-related model has been found to well describe low-frequency $\sigma(\omega)$ behavior. ${ }^{11}$ It is thus plausible to identify an SCPE element, with $1-\gamma_{\mathrm{SC}}$ of the order of $10^{-3}$ to $10^{-4}$, as representing a nearly complete blocking situation, and indeed one often finds values of $A_{\mathrm{SC}}$ consistent with expected double-layer capacitance. Future experiments with a range of electrode separations, allowing 
extrapolation to infinite sample thickness, and/or ones with parent-ion electrodes would certainly be worthwhile.

The K1S-model results shown in column 5 of the table were obtained by using the LEVM program to fit the three column $3 E_{\mathrm{PC}}=0.06-\mathrm{eV}$ PK1-model data sets with the K1S model. To do so, $\beta_{1}$ was held constant at 0.35 , as was 1 $-\gamma_{\mathrm{PC}}=\gamma_{\mathrm{SC}}=0.9999$. Thus, real-part fitting involved the three free parameters $\rho_{0}, \tau_{o}$, and $A_{\mathrm{SC}}$. The fitting yielded exceptionally close agreement, with the fit $S_{\mathrm{F}}$ values increasing from $1.5 \times 10^{-5}$ at 127 to $4.7 \times 10^{-5}$ at $183 \mathrm{~K}$. The resulting $A_{\mathrm{SC}}$ estimates are listed in the table. They led to $E_{\mathrm{SC}} \simeq 0.077 \mathrm{eV}$, but the $5.6 \%$ relative standard deviation of the fit was poor. Although the column 5 results are those for the K1S model, they are identified as involving the $E_{\mathrm{PC}}$ $=0.06-\mathrm{eV}$ PK1 quantity since this value was the starting point for generating the K1S data.

The method of determining $\omega_{\mathrm{CO}}$ was different for the K1S situation from that described above for the PK1 one. The equation $\sigma^{\prime}\left(\omega_{\mathrm{CO}}\right)=r \sigma_{\mathrm{PC}}^{\prime}\left(\omega_{\mathrm{CO}}\right)$ involved the full K1S response on the left side but $\sigma_{\mathrm{SC}}^{\prime}\left(\omega_{\mathrm{CO}}\right)$ could not be used on the right because of the series combination of the model. Instead, at a given temperature the $\mathrm{K} 1 \mathrm{~S} \sigma^{\prime}(\omega)$ data values were fitted with the PK1 model and the resulting PCPE $\sigma_{\mathrm{PC}}^{\prime}(\omega)$ response was used on the right side of the above equation. Once an estimate of $\omega_{\mathrm{CO}}$ was obtained, the full K1S model could be used to obtain the associated $\sigma^{\prime}\left(\omega_{\mathrm{CO}}\right)$ value.

Because the series model of column 5 fits the parallel model so well, one obtains very closely the same crossover results as those for column 3. But notice that the $A_{\mathrm{SC}}$ estimates are not well fitted by either the thermally activated formula, since $S_{\mathrm{FE}} \simeq 5.6 \%$, or by the exponential one involving $b_{\mathrm{PC}}$, where $S_{\mathrm{Fb}} \simeq 10.2 \%$. In addition, the estimates obtained for the $\rho_{0}$ and $\tau_{o}$ parameters were very much smaller than those used for the PK1 model and, even more interesting, they led to estimates of $E_{\tau}$ and $E_{\sigma T}$ of only 0.024 \pm 0.003 and $0.055 \pm 0.007 \mathrm{eV}$, respectively. These estimates are much smaller than the $0.4-\mathrm{eV}$ value used for these quantities in the PK1 model, and Arrhenius response was only approximate since the $S_{\mathrm{F}}$ relative standard deviations of the fits were about 0.056 and 0.152 , respectively.

Another K1S-model fitting, starting with $E_{\mathrm{PC}}=0.09 \mathrm{eV}$, led to even more peculiar results. The K1S data were obtained by the same procedure described above except that the values of $\rho_{0}$ were not taken to be free to vary but were held fixed at the corresponding PK1 values. Although the K1S fits of the PK1 data were nearly as good as those obtained with free $\rho_{0}$, it turned out that all three $\tau_{o}$ estimates were larger than the PK1 ones by a factor of 1.219 ; thus both $\rho_{0} / T$ and $\tau_{o}$ were of thermally activated form with the activation enthalpy of $0.4 \mathrm{eV}$, as before. Although the values of $A_{\mathrm{SC}}$ were much larger than those shown in column 5 of Table I, they were thermally activated with the exact value $E_{\mathrm{SC}}$ $=0.09 \mathrm{eV}$.

These data sets led to the surprising estimates $E_{\omega \mathrm{CO}}$ $=0.696 \pm 0.002$ and $E_{\sigma \mathrm{CO}}=0.599 \pm 0.005 \mathrm{eV}$, with corresponding fit relative standard deviations of 0.034 and 0.104 , respectively. Further, when $A_{\mathrm{SC}}$ was taken to be temperature independent, a negative value of the $\Delta$ quantity of Eq. (3) was found. These results suggest that for the series model, $E_{\sigma \mathrm{CO}}$ depends delicately on the temperature dependence of $A_{\mathrm{SC}}$ and may be either less than or greater than $E_{\sigma T}$, here 0.4 $\mathrm{eV}$, unlike more reasonable PK1-model response.

\section{DISCUSSION OF EVIDENCE FOR ALTERNATE NCL MODELS}

\section{A. The serial approach of León, Rivera, and co-authors}

The work of Refs. 2 and 6 is interpreted by their authors as precluding the use of the parallel composite response model of the present Eq. (1). As they point out, such a sum model implies that NCL effects persist to frequencies much lower than that associated with the residence time of ions in potential wells, a possibility they reject. They therefore propose, in qualitative terms, a serial approach to explain their data. No quantitative model that instantiates the serial model and can be used for data fitting, such as the present composite PK1 model, is provided, however.

This serial model differs from the present composite ones in the following respect. It is assumed that high-frequency NCL (or CL) effects are associated entirely with the vibrational behavior of ions that remain caged within their sites and that there is an abrupt crossover from such localized behavior to hopping to adjacent sites. In the PK1 model, as in the serial one, virtually all ions are vibrating in cages at the highest frequencies. Further, even though the present data analysis using the PK1 model shows the presence of a thermally activated crossover of approximate Arrhenius character, the proportion of ions that stop vibrating and begin hopping increases monotonically as the frequency is lowered. A plausible explanation for such behavior is provided in the following section.

The important conclusion (iv) of Refs. 2 and 6 states that the crossover activation energy found, $E_{\sigma \mathrm{CO}}=0.17$ $\pm 0.03 \mathrm{eV}$, is the same as that obtained from spin-lattice and electrical conductivity relaxation. ${ }^{23}$ In fact, the spin-lattice value quoted in Ref. 23 is $0.15 \mathrm{eV}$, equal to the above value within one standard deviation. Note that this value is very close to that obtained from the original Ngai coupling-model expression, ${ }^{13,22,24} E_{e}=(1-n) E_{\sigma T}$, with $(1-n)=\beta_{1}=0.35$, the present K1-model value. Here $E_{e}$ is the activation energy of the high-frequency-limiting primitive Debye-response part of the Ngai coupling model; see below. In turn, $0.17 \mathrm{eV}$ is identified as the height of a single-ion potential well $E_{m}$, although such an important identification does not appear in Ref. 23. While this conclusion is consonant with the NCL-CL physical model proposed in Refs. 2 and 6, it does not seem conclusively established.

For example, the Ngai coupling model identifies the single-ion energy barrier as that associated with a thermally activated primitive relaxation time $\tau_{e}$, that of the limiting Debye response appearing at and above a temperatureindependent frequency $\omega_{c} \equiv 1 / t_{c}$, of about $10^{12} \mathrm{r} / \mathrm{s}$. Such an identification seems incompatible with the crossover energy $E_{\sigma \mathrm{CO}}$ identification, since NCL behavior is quite different from Debye response and is generally observed at far lower frequencies than $\omega_{c}$. If indeed $E_{\sigma \mathrm{CO}}$ is not the single-ion 
potential barrier, then the serial approach becomes less justifiable. In addition to identifying $E_{\sigma \mathrm{CO}}$ as the single-ion potential, the authors of Refs. 2 and 6 also point out that the $E_{\sigma}$ activation energy of their data equals $0.17 \mathrm{eV}$ at high temperatures and $0.4 \mathrm{eV}$ at low ones, a type of non-Arrhenius behavior.

Although coupling-model explanations of the appearance of a lower-activation-energy response region at sufficiently high temperatures have been provided by Ngai, ${ }^{13,25}$ the cutoff model ${ }^{22}$ provides a possibly superior alternative explanation. This model, involving a short-time cutoff in the K1model distribution of relaxation times, can also lead to such non-Arrhenius behavior. Further, work in progress of the author using the cutoff and coupling models shows that the smaller high-temperature activation energy is neither entirely constant nor necessarily equal to the single-ion activation energy and does not satisfy the $E_{m}=E_{e}=(1-n) E_{\sigma T}$ relation. The above considerations suggest that the (i) and (iv) conclusions of the authors of Refs. 2 and 6, while involving interesting and suggestive hypotheses, are currently unproven.

\section{B. The PCPE bulk-dielectric NCL coupling model}

The present $b T$ results of column 6 of Table I, shown by the open circles in Fig. 1, as well as the experimental results of Fig. 2(b) of Ref. 6, do not lie closely on a straight line. Further, even when the NCL parts of the response are taken of Arrhenius form, for the present virtually exact results the crossover points are not exactly of such character but involve a relative standard deviation of fit, given by $S_{\mathrm{F} \sigma \mathrm{C}} \cong S_{\mathrm{F} \omega \mathrm{C}}$ of about 4\%, as indicated in Table I. Finally, it should be mentioned that in order for the present PK1 or K1S models to yield a value of $E_{\sigma \mathrm{CO}}$ of $0.17 \mathrm{eV}$, an appreciably larger value of $b$ is required than that found in Ref. 6 .

If the serial approach of Refs. 2 and 6 were to be rejected as unlikely and unproven, what other explanation of NCL behavior might be more plausible? Recent complexnonlinear-least-squares fitting of $x_{c} \mathrm{Na}_{2} \mathrm{O} \cdot\left(1-x_{c}\right) \mathrm{GeO}_{2}$ data over an appreciable range of ionic concentrations, $x_{c}$ has strongly suggested that NCL effects were present and could be well represented by a PCPE element. ${ }^{4}$ This response, being in parallel with conductive-system $\mathrm{K} 1$ response at the complex conductivity level, may be identified as part of the bulk-dielectric response, that involving the high-frequency bulk-dielectric constant $\varepsilon_{D_{\infty}}$, and it causes $\varepsilon_{D_{\infty}}$, when taken as a free fitting parameter, to increase with increasing $x_{c}$.
This identification is indeed appropriate because all ionichopping effects were well fitted by the K1 model alone, ${ }^{4}$ thus implying the need for an additional nonionic process to account for NCL behavior.

In Ref. 4 it is found that in the $x_{c} \rightarrow 0$ limit one may define the $\varepsilon_{D \infty 0}$ dielectric constant quantity, appropriate in the absence of alkali material. Rather than model the increase of the bulk-dielectric constant and NCL behavior by the free parameter $\varepsilon_{D \infty}$ alone (no NCL) or by PCPE response alone ( $\varepsilon_{D \infty}$ effects roughly included as part of the CPE), good fits over an appreciable range of concentrations were found by taking $\varepsilon_{D \infty}$ fixed at $\varepsilon_{D \infty 0}$ and then including in the full response model a PCPE representing the power-law incremental increase in the effective $\varepsilon_{D \infty}$ and associated NCL behavior.

In this NCL model it is assumed that the charge-carrier motion, approximately vibratory and dipolarlike at high frequencies, as assumed in conclusion (v), and involving both forward and some reverse hops at lower ones, ${ }^{26}$ is coupled to the dipolar response of the bulk material in such a way that increases in ionic concentration increase the effective highfrequency-limiting bulk-dielectric constant, just as found from accurate fitting results. ${ }^{4}$ In turn, such increased coupling would be expected to increase the PCPE $A_{\mathrm{PC}} \mathrm{NCL}$ parameter, again just as observed. In this NCL model, unlike the serial one, ions thus play an indirect rather than a direct role in leading to NCL behavior. Clearly, theoretical analysis of this ion-dipole coupling model is needed for comparison with the quantitative results presented in Ref. 4.

Finally, note that there is another independent effect that might lead to changes in $\varepsilon_{D \infty}$ as $x_{c}$ increases. It would arise from any difference in the polarizability of the $x_{c}$ part of the bulk material as compared to that of the $1-x_{c}$ part. It is likely to be small in any event and has not been accounted for in the analysis of Ref. 4.

In conclusion, the present results indicate that there seems no need to invoke a serial model to explain the important crossover behavior described in Refs. 2 and 6, as in conclusions (i) and (v). Further, conclusions (ii)-(iv) seem to be inappropriate, inaccurate, and/or unlikely.

\section{ACKNOWLEDGMENT}

It is a pleasure to thank Dr. Carlos León for providing a preprint of the present Ref. 6 and for stimulating and valuable correspondence.
*Email address: macd@email.unc.edu

${ }^{1}$ K. L. Ngai, J. Chem. Phys. 110, 10576 (1999).

${ }^{2}$ C. León, A. Rivera, A. Várez, J. Sanz, J. Santamaria, and K. L. Ngai, Phys. Rev. Lett. 86, 1279 (2001).

${ }^{3}$ J. R. Macdonald, J. Chem. Phys. 115, 6192 (2001).

${ }^{4}$ J. R. Macdonald, J. Chem. Phys. 116, 3401 (2002).

${ }^{5}$ J. R. Macdonald, J. Non-Cryst. Solids 307-310, 913 (2002).

${ }^{6}$ A. Rivera, C. León, J. Sanz, J. Santamaria, C. T. Moynihan, and K. L. Ngai, Phys. Rev. B 65, 224302 (2002).

${ }^{7}$ W. K. Lee, J. F. Liu, and A. S. Nowick, Phys. Rev. Lett. 67, 1559
(1991).

${ }^{8}$ B. S. Lim, A. V. Vaysleyb, and A. S. Nowick, Appl. Phys. A: Solids Surf. 56, 8 (1993).

${ }^{9}$ A. S. Nowick, B. S. Lim, and A. V. Vaysleyb, J. Non-Cryst. Solids 172-174, 1243 (1994).

${ }^{10}$ J. R. Macdonald, Solid State Ionics 133, 79 (2000).

${ }^{11}$ J. R. Macdonald, J. Non-Cryst. Solids 210, 70 (1997).

${ }^{12}$ Impedance Spectroscopy-Emphasizing Solid Materials and Systems, edited by J. R. Macdonald (Wiley-Interscience, New York, 1987). 
${ }^{13}$ K. L. Ngai, Philos. Mag. B 77, 187 (1998).

${ }^{14}$ C. Cramer, K. Funke, and T. Saatkamp, Philos. Mag. B 71, 701 (1995).

${ }^{15}$ J. R. Macdonald and L. D. Potter, Jr., Solid State Ionics 23, 61 (1987); J. R. Macdonald, J. Comput. Phys. 157, 280 (2000). The newest version of the comprehensive LEVM complex-nonlinearleast-squares-fitting program may be downloaded at no cost from http://www.physics.unc.edu/ macd. It includes an extensive manual, executable programs, and full source code. More information is provided about LEVM at this web address.

${ }^{16}$ A. S. Nowick, A. V. Vaysleyb, and W. Liu, Solid State Ionics $\mathbf{1 0 5}$, 121 (1998).

${ }^{17}$ K. L. Ngai, J. Non-Cryst. Solids 203, 232 (1996).
${ }^{18}$ J. R. Macdonald, J. Appl. Phys. 82, 3962 (1997).

${ }^{19}$ J. R. Macdonald, Phys. Rev. B 63, 052205 (2001).

${ }^{20}$ J. R. Macdonald, J. Appl. Phys. 90, 153 (2001). In Eq. (10), $\sigma_{0} \Gamma$ should be replaced by $\sigma_{0} \tau_{o} \Gamma$.

${ }^{21}$ J. R. Macdonald, Solid State Ionics (to be published).

${ }^{22}$ J. R. Macdonald, J. Appl. Phys. 84, 812 (1998).

${ }^{23}$ C. León, J. Santamaria, M. A. Paris, J. Sanz, J. Ibarra, and L. M. Torre, Phys. Rev. B 56, 5302 (1997).

${ }^{24}$ K. L. Ngai and O. Kanert, Solid State Ionics 53-56, 936 (1992); K. L. Ngai, J. Chem. Phys. 98, 6424 (1993).

${ }^{25}$ K. L. Ngai and A. K. Rizos, Phys. Rev. Lett. 76, 1296 (1996).

${ }^{26}$ C. León, M. L. Lucia, and J. Santamaria, Phys. Rev. B 55, 882 (1997). 